\title{
Performance indicators for reactive distillation design
}

\author{
Rui M. Filipe ${ }^{1 *}$, Henrique A. Matos ${ }^{2}$, Augusto Q. Novais ${ }^{3}$ \\ ${ }^{1}$ Departamento de Engenharia Química, Instituto Superior de Engenharia de Lisboa, \\ R. Conselheiro Emídio Navarro, 1, 1959-007 Lisboa, Portugal. \\ ${ }^{2}$ Centro Processos Químicos, Departamento de Engenharia Química e Biológica, Instituto \\ Superior Técnico, Av. Rovisco Pais, 1049-001 Lisboa, Portugal. \\ ${ }^{3}$ Departamento de Modelação e Simulação de Processos, Instituto Nacional de Engenharia, \\ Tecnologia e Inovação, Est. do Paço do Lumiar, 1649-038 Lisboa, Portugal.
}

Keywords: Reactive distillation, multi-objective optimization, feed quality

Topic: Systematic methods and tools for managing the complexity

\begin{abstract}
A cost indicator for the design and multi-objective optimization of reactive distillation columns, designated capacity, was introduced in previous work by the authors. The question of this indicator's effectiveness as a measure of the actual column cost, is herein investigated over a number of designs by comparing it with the value obtained by means of conventional costing procedures. The results show that the level of accuracy obtained when using capacity is satisfactory and certainly acceptable for a preliminary design stage.
\end{abstract}

\section{Introduction}

Reactive distillation (RD) combines reaction and separation into the same physical shell. This combination can lead to both economic and environmental gains resulting from process intensification (Malone and Doherty, 2000; Taylor and Krishna, 2000) and therefore to systems with significantly greener engineering attributes (Malone et al., 2003).

However the design of these systems can prove very complex and costly. Design should therefore be preceded by preliminary studies to assess first the potential adequacy of the technology. With this aim some work was developed to create a framework combining feasible regions and optimization techniques to the design and multi-objective optimization of complex reactive distillation columns, for reacting systems with variable degrees of relative volatilities (Filipe et al., 2006; 2007; 2008b).

This led to the consideration of RD columns with distributed feeds, with many of them involving the combination of superheated and subcooled feeds. This combination provides a source or a sink of heat at specified trays of the columns, which favors reaction while reducing the total reactive holdup requirements.

To assess the impact of employing such combined feeds, a cost indicator, designated capacity, was introduced as a novel optimization objective criterion (Filipe et al., 2006). This cost indicator, based on the capacity variables (Jobson et al., 1996), was defined as the weighted sum of liquid and vapor internal flows divided by the total feed to the column.

Together with the total number of stages and reactive holdup this indicator was used to build the Pareto front that contains the efficient set of solutions. Considering the very large number of designs involved in the evaluation, an indicator was preferred to a detailed cost calculation.

Following a strategy of refining the Pareto optimal designs, some aspects related with the practical implementation of a few promising designs were investigated (Filipe et al., 2008a) by means of a number of sensitivity tests on catalyst usage and feed quality. The advantage

\footnotetext{
* Corresponding author. Tel + 351218317 000. E-mail: rfilipe@isel.ipl.pt
} 
of using combined feeds was confirmed, as well as the need for a careful selection of the feed qualities.

The capacity indicator proved valuable as an optimization criterion, not only because it is efficient in comparing thousands of different designs, but also because it effectively limits the range of the feed qualities used. However, some drawbacks have been identified in its formulation, namely it makes no distinction between reactive and non reactive trays and the RD effective capital and operational costs are not explicitly shown.

This work presents a study where the effectiveness of the proposed cost indicator is assessed and, in addition, the optimal solutions identified in previous stages of the design procedure are tested at an advanced level. The size (height and diameter) of the column, energy and catalyst demands are quantified in the Aspen Plus detailed model of some designs and the total cost of the column estimated using common cost correlations. These values can be correlated with the corresponding capacity values, thus allowing the estimation of new weights for the factors present in the initial capacity definition.

As an illustrative example, a system with ideal vapor liquid behavior is employed, i.e., the olefin metathesis system (wherein 2-pentene reacts to form 2-butene and 3-hexene). For this particular reactive system, the reactant boiling point falls between those of the two products, allowing for a wide range of feasible column designs.

\section{Methodology}

To develop this study, six different solutions belonging to the Pareto front relating reactive and capacity (cost indicator) previously generated (Filipe et al., 2008b) were selected. The main assumptions for the optimization methodology developed in GAMS to achieve the Pareto fronts are: steady state operation, constant pressure, vapor-liquid equilibrium at every stage, kinetically controlled reaction occurring in the liquid phase, and negligible heat effects. For each design, the methodology applied determines the optimal locations for the catalyst, feeds and feed quality.

The selected set of solutions is used to initialize simulations in Aspen Plus, using the RadFrac model and the Ideal property method. All the design specifications, such as the number of stages, reboil ratio, distillate to feed ratio, location and quality of the feeds, as well as the reactive holdup distribution, are taken from the former optimization results. Aspen Plus does not support the direct specification of the feed quality. To overcome this, a design specification is implemented: the feed temperature is adjusted to provide the required energy for a change in the internal liquid flow according to the equation $L_{n}=L_{n+1}+q \cdot F$, where $F$ is the feed flow, $q$ the feed quality, and $L_{n+1}$ and $L_{n}$ the liquid entering and leaving the tray, respectively.

The capacity cost indicator previously defined (Filipe et al., 2006) is based on the size of internal flows, feeds and number of stages, providing an expedient method for the evaluation of a large number of solutions. Equation 1 shows how to calculate the capacity for a column with $\mathrm{N}$ stages, where $\mathrm{i}=1$ refers to the condenser and $\mathrm{i}=\mathrm{N}$ to the reboiler, $\mathrm{V}_{2}$ is the vapor condensed at the total condenser, $B$ is the bottom liquid flow and $w_{B}$ and $w_{C}$ are the weights for the boiling and condensing capacity, respectively. Both weights were set to unity during the optimization step.

$$
\text { Capacity }=\frac{w_{B}\left(\sum_{i=2}^{N} V_{i}\right)+w_{C}\left(\sum_{i=2}^{N-1} L_{i}+V_{2}+B\right)}{\sum_{i=1}^{N} F_{i}}
$$

Eq. 1

As many reported designs tend to be operationally unrealistic (e.g. very large number of feeds, too large or too small feed temperatures), some modifications of the optimal designs previously obtained were considered. Three different scenarios, A, B and C are defined by 
modifying the original optimal design, for each of the selected 6 cases. In scenario $A$, the number of feeds is limited to two, while maintaining the amount of energy supplied by the feed streams to the column. Scenario B considers a more realistic adaptation, where the number of feeds is reduced to two and the feed quality value is bounded by an upper temperature of $650 \mathrm{~K}$ and a lower of $298 \mathrm{~K}$. In this same scenario, the cooling requirements of the cold stream are reduced to zero. Moreover the elimination of unrealistic temperature conditions by increasing the temperature of the cold feeds, has only a slight effect on product purity as demonstrated previously (Filipe et al., 2008a). Scenario $C$ represents a departing approach from the previous ones, where the advantages of using subcooled and superheated feeds are not considered. Instead, feed conditions - dew and boiling points of the feed stream - usually found in industrially operating columns are assumed. The number of feeds is also reduced to two in this scenario and the behavior of the capacity is tested for a diverse range of feed qualities.

The total annual cost (TAC) is calculated as the sum of the annual capital cost and energy cost, using the correlations reported by Luyben (2000). Annual capital cost is assumed to be the capital investment divided by a payback period of 3 years. The column shell, trays, reboiler, condenser, heat exchangers for feeds and corresponding heating utilities, are considered in the calculations. The cost of the catalyst is not considered, as the capacity is supposed to include only capital and utilities cost, but it is accounted for in the multi-objective optimization methodology, in terms of the reactive holdup variable.

The olefin metathesis system is used, with the physical properties and reaction kinetics being taken from the literature (Okasinski and Doherty, 1998). The reaction is considered only to occur in the liquid phase with a negligible heat of reaction and ideal vapor-liquid equilibrium behavior at atmospheric pressure. The specifications for column operation are taken from Hoffmaster and Hauan (2006), where the goal is to convert a pure 2-pentene feed into product streams of 2-butene and 3-hexene with a purity of at least 98 mole percent using a feed flow of $2 \mathrm{kmol} / \mathrm{h}$ and a distillate to feed ratio of 0.5 .

\section{Results and discussion}

The details for the selected cases are shown in Table 1 . The number of stages ranges between 29 and 16, the reactive holdup between 3.2 and 8 and the capacity between 90.4 and 41. As discussed earlier (Filipe et al., 2008b) the capacity increases with the number of stages (higher internal flows in larger columns) and, for the same design specification, higher product purities require higher capacities (increased residence time in the reactive trays). Column diameter changes only slightly within each scenario, while between scenarios a significant reduction is observed in $C$ when compared to $A$ and $B$, which is justified by its lower internal flows due to the feed qualities used.

Although the design specifications used in the optimization methodology required a purity of $98 \%$ in the products, the assumptions made at the optimization stage and the more rigorous calculations employed by Aspen Plus lead to different purities. In addition, the changes in the feed qualities also contribute to this effect. Product purity is greatly reduced in scenario C, especially in the columns with larger number of stages, where the reduction in the internal flows is more noticeable. This reduction in the internal flows, also revealed by the reduction in capacity, decreases the residence time in the reactive trays, thus deteriorating the specifications of the products.

Figure 1 depicts the variation of product purity with capacity for scenarios A and $\mathrm{B}$. The dashed line indicates the specification used in the optimization methodology. It is noticeable that the adjustments made in the feed qualities increase the product purities in most of the designs at the expense of a penalty in capacity, which is always increased. This is a somewhat expected result as these scenarios are based in optimal designs where the reactive holdup and capacity had been minimized. In addition, contrary to the observed effect 
for a single column, capacity is found not to increase with product purity while comparing different columns, since they correspond to different performance levels.

\section{Table 1 - Case details}

\begin{tabular}{|c|c|c|c|c|c|c|c|c|}
\hline & & Case & 1 & 2 & 3 & 4 & 5 & 6 \\
\hline \multirow{7}{*}{ 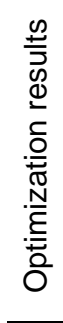 } & & $\mathrm{RH}$ & 3.2 & 3.5 & 4.2 & 5.2 & 6.4 & 8.0 \\
\hline & & Cap & 90.4 & 70.7 & 54.6 & 46.8 & 43.4 & 41.0 \\
\hline & & NT & 29 & 25 & 19 & 18 & 17 & 16 \\
\hline & & NF & 3 & 5 & 3 & 3 & 3 & 3 \\
\hline & & FT & $9,10,21$ & $9,10,15,16,17$ & $9,11,12$ & $9,10,11$ & $8,9,10$ & $8,9,10$ \\
\hline & & $F(\%)$ & $28,26,46$ & $12,45,9,15,19$ & $60,18,22$ & $63,14,23$ & $29,42,29$ & $46,38,16$ \\
\hline & & $q$ & $2,2,-2$ & $2,2,-2,-2,-2$ & $2,-2,-2$ & $2,-2,-2$ & $2,1.3,-2$ & $2,0,-2$ \\
\hline \multirow{23}{*}{ 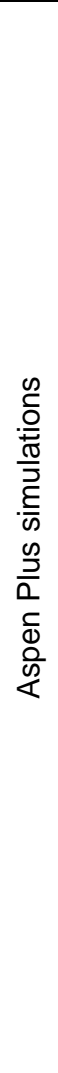 } & Scenario & FT & 9,21 & 9,17 & 9,12 & 9,11 & 8,9 & 8,10 \\
\hline & & $F(\%)$ & 55,45 & 58,42 & 60,40 & 62,38 & 70,30 & 46,54 \\
\hline & A & $q$ & $2,-2$ & $2,-2$ & $2,-2$ & $2,-2$ & $1.6,-2$ & $2,-0.6$ \\
\hline & & Cap & 96.6 & 78.2 & 62.9 & 49.9 & 45.5 & 42.5 \\
\hline & & $\mathrm{D}_{\mathrm{col}}$ & 0.18 & 0.18 & 0.19 & 0.18 & 0.17 & 0.17 \\
\hline & & Purity (\%) & 97.55 & 97.92 & 98.06 & 97.66 & 97.70 & 97.21 \\
\hline & & Equipment (\$) & 58317 & 53367 & 50046 & 46071 & 42329 & 41442 \\
\hline & & Utilities (\$) & 5950 & 5860 & 6192 & 5884 & 5214 & 5257 \\
\hline & & TAC (\$) & 25389 & 23649 & 22874 & 21241 & 19323 & 19071 \\
\hline & B & $q$ & $1,-1.7$ & $1,-1.7$ & $0.98,-1.7$ & $0.96,-1.8$ & $0.97,-1.8$ & $0.91,-1.7$ \\
\hline & & Cap & 100.1 & 83.7 & 66.5 & 59.2 & 51.3 & 49.8 \\
\hline & & $\mathrm{D}_{\mathrm{col}}$ & 0.18 & 0.17 & 0.18 & 0.18 & 0.17 & 0.17 \\
\hline & & Purity (\%) & 97.14 & 97.98 & 98.20 & 98.35 & 98.23 & 97.83 \\
\hline & & Equipment (\$) & 55022 & 50718 & 47241 & 44806 & 40994 & 42335 \\
\hline & & Utilities (\$) & 11110 & 10983 & 11751 & 11175 & 9965 & 10375 \\
\hline & & TAC (\$) & 29451 & 27888 & 27498 & 26111 & 23630 & 24486 \\
\hline & $\mathrm{C}$ & $q$ & 1,0 & 1,0 & 1,0 & 1,0 & 1,0 & 1,0 \\
\hline & & Cap & 66.4 & 58.6 & 50.3 & 45.5 & 41.7 & 44.1 \\
\hline & & $\mathrm{D}_{\mathrm{col}}(\mathrm{m})$ & 0.14 & 0.14 & 0.15 & 0.14 & 0.14 & 0.15 \\
\hline & & Purity (\%) & 82.19 & 88.14 & 94.97 & 96.10 & 96.37 & 97.06 \\
\hline & & Equipment (\$) & 43596 & 41166 & 39646 & 37755 & 35556 & 39268 \\
\hline & & Utilities (\$) & 6832 & 7009 & 8001 & 7665 & 7244 & 8719 \\
\hline & & TAC (\$) & 21364 & 20731 & 21216 & 20250 & 19096 & 21808 \\
\hline
\end{tabular}

NT number of trays, NF number of feeds, FT feed tray, F amount of feed (\%), q feed quality, Cap capacity, $D_{\text {col }}$ column diameter, TAC total annualized costs.

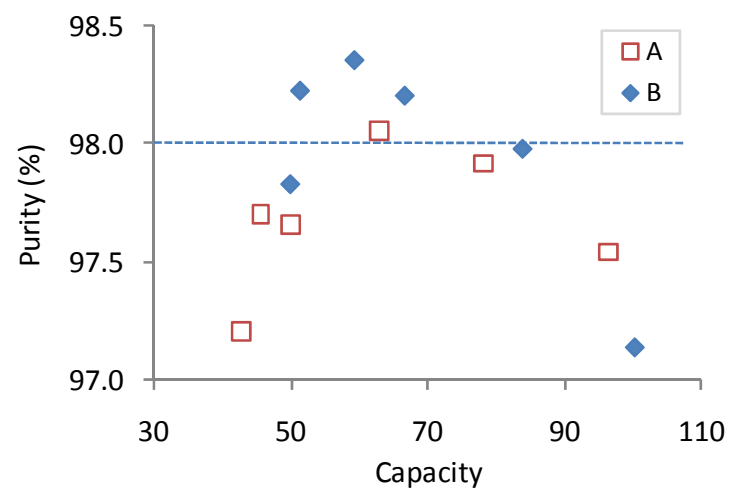

Figure 1 - Purity versus capacity for scenarios A and B 


\subsection{Comparison of capacity with total annualized costs}

In Figure 2, the capacity, and the equipment and utilities costs are depicted for scenario B. All these variables decrease as the height of the column decreases (a high case number indicates a low number of stages). It is noticeable that capacity describes correctly the cost trend, while displaying greater sensitivity at the upper end of the cost curves.

In Figure 3 the variation of the total annual costs and capacity for case B are normalized, by dividing the value of each case by the average value of the six cases. It now becomes clearer that the deviation occurs at both ends of the cost curves. The calculated mean deviation is about $20 \%$, thus capacity may be considered an acceptable cost indicator for an early stage conceptual process design. It is also advantageous when the design is not yet fully specified as only the knowledge of internal flows and feed flows is required. In addition, its calculation is straightforward, making it adequate for the comparison of very large number of designs.

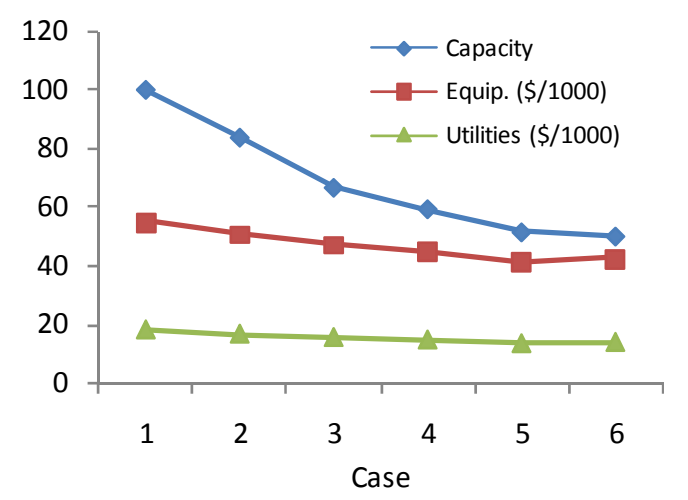

Figure 2 - Capacity, equipment and utilities cost for scenario $B$

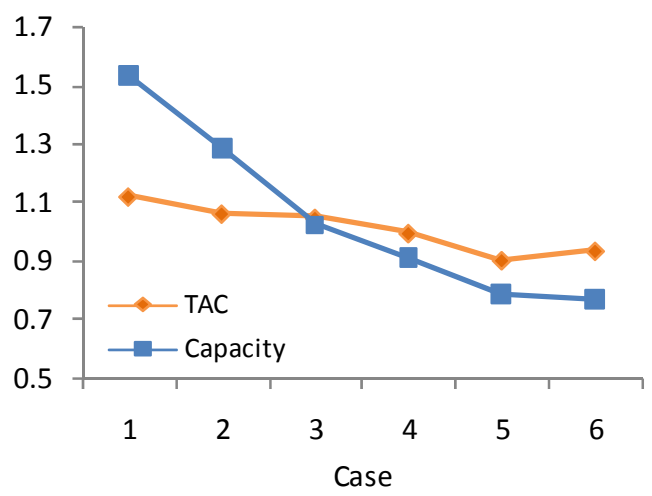

Figure 3 - Normalized capacity and TAC for scenario B

\subsection{Capacity weights}

The effect of weights $w_{C}$ and $w_{B}$ on the capacity cost indicator was assessed and it is concluded that no significant effects were noticed. The values for the individual boiling and condensing capacities (Figure 4) typically differ less than $5 \%$, so the assumed values of $w_{B}=w_{C}=1$, employed for the present study seem justified.

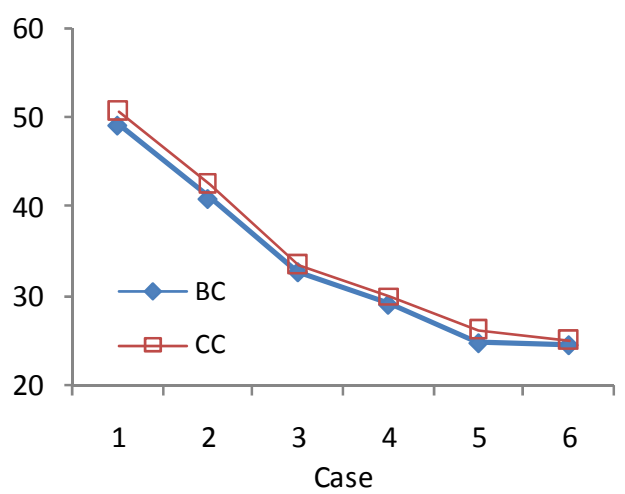

Figure 4 - Boiling capacity (BC) and condensing capacity(CC) for scenario B

\section{Conclusions}

This paper addresses the use of performance indicators for the design of reactive distillation columns. Specifically, the analysis of a cost indicator previously used in the classification and selection of several thousands of preliminary designs is addressed. The total annualized 
costs are calculated and compared to capacity indicator values for several different designs derived from the optimal designs previously obtained from optimization runs performed in GAMS. The results show that capacity and costs have a common trend, within an average error of $20 \%$, pointing to capacity as a suitable indicator for preliminary design analysis.

The influence of the weights used in the definition of capacity was also assessed with a view of improving its accuracy, but no significant improvements could be achieved as the individual capacity values are quite close.

On-going work aims to establish the effect of the weights during the optimization process and to further sophisticate the capacity indicator with a view to make it more selective in terms of the number and characteristics of the optimal preliminary designs.

\section{References}

Filipe, R.M., Novais, A.Q., Hauan, S. (2006). Multi-objective optimization of reactive distillation columns using feasible regions. $17^{\text {th }}$ International Congress of Chemical and Process Engineering, Prague, Czech Republic.

Filipe, R.M., Hauan, S., Matos, H.A., Novais, A.Q. (2007). Multi-Objective Design of Reactive Distillation. $17^{\text {th }}$ European Symposium on Computer Aided Process Engineering. V. Plesu and P. S. Agachi. 24: 407-412.

Filipe, R.M., Hauan, S., Matos, H.A., Novais, A.Q. (2008a). A sensitivity analysis on optimal solutions obtained for a reactive distillation colum. $18^{\text {th }}$ European Symposium on Computer Aided Process Engineering. B. Braunschweig and X. Joulia. Lyon, France. 25: 211-216.

Filipe, R.M., Turnberg, S., Hauan, S., Matos, H.A., Novais, A.Q. (2008b). Multi-objective design of reactive distillation with feasible regions. Industrial \& Engineering Chemistry Research, (accepted for publication).

Hoffmaster, W.R., Hauan, S. (2006). Using feasible regions to design and optimize reactive distillation columns with ideal VLE. AIChE Journal, 52, 1744-1753.

Jobson, M., Hildebrandt, D., Glasser, D. (1996). Variables indicating the cost of vapour-liquid equilibrium separation processes. Chemical Engineering Science, 51, 4749-4757.

Luyben, W.L. (2000). Economic and Dynamic Impact of the Use of Excess Reactant in Reactive Distillation Systems. Industrial \& Engineering Chemistry Research, 39, 2935-2946.

Malone, M.F., Doherty, M.F. (2000). Reactive Distillation. Industrial \& Engineering Chemistry Research, 39, 3953-3957.

Malone, M.F., Huss, R.S., Doherty, M.F. (2003). Green chemical engineering aspects of reactive distillation. Environmental Science \& Technology, 37, 5325-5329.

Okasinski, M.J., Doherty, M.F. (1998). Design Method for Kinetically Controlled, Staged Reactive Distillation Columns. Industrial \& Engineering Chemistry Research, 37, 2821-2834.

Taylor, R., Krishna, R. (2000). Modelling Reactive Distillation. Chemical Engineering Science, 55, 5183-5229. 